\title{
ON THE DIMENSION OF LIMITS OF INVERSE SYSTEMS
}

\author{
YUKINOBU YAJIMA
}

\begin{abstract}
We say that the limit of an inverse system $X=\varliminf_{i m}\left\{X_{\lambda}, \pi_{\mu}^{\lambda}, \Lambda\right\}$ is cylindrical if each finite cozero cover of $X$ has a $\sigma$-locally finite refinement consisting of sets of the form $\pi_{\lambda}^{-1}(U)$, where $U$ is a cozero-set in $X_{\lambda}$ and $\pi_{\lambda}: X \rightarrow X_{\lambda}$ is the projection.

We prove that if $X$ is cylindrical, then $\operatorname{dim} X=\sup \left\{\operatorname{dim} X_{\lambda}: \lambda \in \Lambda\right\}$.
\end{abstract}

1. Introduction. We give a sufficient condition for the limit of an inverse system of topological spaces with the covering dimension $\leq n$ to have the dimension $\leq n$.

We say that the limit of an inverse system $X=\varliminf_{i}\left\{X_{\lambda}, \pi_{\mu}^{\lambda}, \Lambda\right\}$ is cylindrical if each finite cozero cover of $X$ has a $\sigma$-locally finite refinement consisting of sets of the form $\pi_{\lambda}^{-1}(U)$, where $U$ is a cozero-set in $X_{\lambda}$ and $\pi_{\lambda}: X \rightarrow X_{\lambda}$ is the projection. In this paper, we prove

"If $X$ is cylindrical, then $\operatorname{dim} X=\sup \left\{\operatorname{dim} X_{\lambda}: \lambda \in \Lambda\right\}$ ".

Next, we show that the limit of an inverse system of paracompact spaces and perfect maps is cylindrical and that the limit of an perforable inverse sequence of normal spaces is cylindrical, thus obtaining earlier results of Katuta-Pasynkov $\left[\mathbf{K}, \mathbf{P}_{\mathbf{1}}\right]$ and Nagami-Pasynkov $\left[\mathbf{N}_{\mathbf{2}}, \mathbf{P}_{\mathbf{2}}\right]$, respectively, as corollaries. Since every Cartesian product of infinitely many spaces is the limit of the inverse system of its finite subproducts, we immediately obtain a sufficient condition for the dimension of the Cartesian product not to exceed the supremum of the dimensions of its finite subproducts. In particular, this condition is satisfied for a Cartesian product of metrizable spaces, thus yielding E. Pol's result [Po] as a corollary.

2. Preliminaries. Throughout this paper, by a space and a map we mean a topological space and a continuous map, respectively. No separation axioms are assumed. However, regular, normal and paracompact spaces are always assumed to be $T_{2}$. For a set $A$, by $|A|$ we mean the cardinality of $A$. Natural numbers are denoted by $i, j, k$ and $m$. The smallest infinite ordinal (cardinal) number is denoted by $\omega\left(\aleph_{0}\right)$.

The dimension dim always means the covering dimension, for which we use a nonnegative integer $n$. For a space $X$, a cozero-set in $X$ is a set of the form $f^{-1}((0,1])$ for some map $f: X \rightarrow[0,1]$.

Let $\left\{X_{\lambda}, \pi_{\mu}^{\lambda}, \Lambda\right\}$ be an inverse system with the limit $X$. Then a map $\pi_{\lambda}: X \rightarrow X_{\lambda}$ means the projection for each $\lambda \in \Lambda$. The directed set $\Lambda$ is omitted if $|\Lambda| \leq \aleph_{0}$.

Received by the editors June 13, 1983 and, in revised form, December 9, 1983.

1980 Mathematics Subject Classification. Primary 54B25, 54F45; Secondary 54B10.

Key words and phrases. Covering dimension, limit of inverse system, cylindrical, cozero cylinder, perforable inverse sequence, Cartesian product, finite subproduct. 
Let $X=\prod_{\lambda \in \Lambda} X_{\lambda}$ be a Cartesian product. For each $\xi \subset \Lambda$, let $X_{\xi}=\prod_{\lambda \in \xi} X_{\lambda}$ and $\pi_{\xi}: X \rightarrow X_{\xi}$ the projection. For each $\xi \subset \Lambda(\Gamma \subset \Lambda)$ with $|\xi|<\aleph_{0}\left(|\Gamma| \leq \aleph_{0}\right)$, the product $X_{\xi}\left(X_{\Gamma}\right)$ is said to be a finite (countable) subproduct of $X$.

3. Main theorem. The following definition is similar to that of rectangularity for finite products in the sense of Pasynkov $\left[\mathbf{P}_{\mathbf{3}}\right]$.

DEFINITION 1. The limit of an inverse system $X=\varliminf_{\longleftarrow}\left\{X_{\lambda}, \pi_{\mu}^{\lambda}, \Lambda\right\}$ is said to be cylindrical, if each finite cozero cover of $X$ has a $\sigma$-locally finite refinement by sets of the form $\pi_{\lambda}^{-1}(U)$, where $\lambda \in \Lambda$ and $U$ is a cozero-set in $X_{\lambda}$. Such a set $\pi_{\lambda}^{-1}(U)$ is said to be a cozero cylinder in $X$.

Our main theorem is as follows.

THEOREM. Let $X=\lim _{\lfloor}\left\{X_{\lambda}, \pi_{\mu}^{\lambda}, \Lambda\right\}$ be the limit of an inverse system of spaces $X_{\lambda}$ with $\operatorname{dim} X_{\lambda} \leq n$. If $X$ is cylindrical, then $\operatorname{dim} X \leq n$.

The idea of the proof of Theorem is essentially due to Pasynkov $\left[\mathbf{P}_{\mathbf{3}}\right.$, Theorem 17]. Indeed, we make use of the following result in $\left[\mathbf{P}_{\mathbf{3}}\right.$, Proposition 10].

LEMMA 1. Suppose that for each finite cozero cover $O$ of a space $X$ there exists an inverse system $\left\{R_{\alpha}, \phi_{\beta}^{\alpha}, A\right\}$ of metrizable spaces $R_{\alpha}$ with $\operatorname{dim} R_{\alpha} \leq n$, satisfying the following:

(a) for each $\alpha \in A$, there exists a map $f_{\alpha}: X \rightarrow R_{\alpha}$ such that $f_{\beta}=\phi_{\beta}^{\alpha} \circ f_{\alpha}$ for $\beta<\alpha$,

(b) there exist some $A_{0} \subset A$ and open sets $V_{\alpha}$ in $R_{\alpha}, \alpha \in A_{0}$, such that $\left\{f_{\alpha}^{-1}\left(V_{\alpha}\right): \alpha \in A_{0}\right\}$ is a $\sigma$-locally finite refinement of 0 .

Then we have $\operatorname{dim} X \leq n$.

Proof of THEOREM. Let $O$ be any finite cozero cover of $X$. By the assumption, it has a $\sigma$-locally finite refinement $\left\{\pi_{\lambda_{\alpha}}^{-1}\left(U_{\alpha}\right): \alpha \in A\right\}$ by cozero cylinders in $X$. For each $\alpha \in A$, we take a map $g_{\alpha}: X_{\lambda_{\alpha}} \rightarrow[0,1]$ such that $g_{\alpha}^{-1}((0,1])=U_{\alpha}$. By Pasynkov's factorization theorem (cf. [ $\mathbf{P}_{\mathbf{3}}$, Theorem 2]), there exist a metrizable space $R_{\alpha}$ with $\operatorname{dim} R_{\alpha} \leq n$, an "onto" map $f_{\alpha}: X_{\lambda_{\alpha}} \rightarrow R_{\alpha}$ and a map $h_{\alpha}: R_{\alpha} \rightarrow[0,1]$ such that $g_{\alpha}=h_{\alpha} \circ f_{\alpha}$ for each $\alpha \in A$. Put $V_{\alpha}=h_{\alpha}^{-1}((0,1])$ and $\tilde{f}_{\alpha}=f_{\alpha} \circ \pi_{\lambda_{\alpha}}$ for each $\alpha \in A$. Then we have

(1) $\tilde{f}_{\alpha}^{-1}\left(V_{\alpha}\right)=\pi_{\lambda_{\alpha}}^{-1}\left(U_{\alpha}\right)$.

Let $A^{*}$ be the set of all finite subsets of $A$. For each $a \in A^{*}$, we construct a metrizable space $R_{a}$ with $\operatorname{dim} R_{a} \leq n$, a $\lambda(a) \in \Lambda$ with $\lambda(a) \geq \lambda(b)$ for $b \subset a$, an "onto" map $f_{a}: X_{\lambda(a)} \rightarrow R_{a}$ and maps $\phi_{b}^{a}: R_{a} \rightarrow R_{b}$ for $b \subset a$, satisfying the following:

(2) $R_{a}=R_{\alpha}, \lambda(a)=\lambda_{\alpha}$ and $f_{a}=f_{\alpha}$ if $a=(\alpha) \in A^{*}$,

(3) $\tilde{f}_{b}=\phi_{b}^{a} \circ \tilde{f}_{a}$ for $b \subset a$, where $\tilde{f}_{a}=f_{a} \circ \pi_{\lambda(a)}$,

(4) $\phi_{c}^{a}=\phi_{c}^{b} \circ \phi_{b}^{a}$ for $c \subset b \subset a$.

For each $a \in A^{*}$ with $|a|=1$, we define $R_{a}, \lambda(a)$ and $f_{a}$ as in (2). Assume that the above construction has been already performed for each $a \in A^{*}$ with $|a|<k$. Consider an $a \in A^{*}$ with $|a|=k$. We can choose a $\lambda(a) \in \Lambda$ with $\lambda(a) \geq \lambda(b)$ for each $b \subsetneq a$. We put $S_{a}=\prod_{b \subsetneq a} R_{b}$. We take the map $g_{a}: X_{\lambda(a)} \rightarrow S_{a}$ defined by $g_{a}(x)=\left(f_{b} \circ \pi_{\lambda(b)}^{\lambda(a)}(x)\right)_{b \subseteq a}$ for each $x \in X_{\lambda(a)}$. Again, by Pasynkov's factorization theorem, there exist a metrizable space $R_{a}$ with $\operatorname{dim} R_{a} \leq n$, an "onto" map $f_{a}: X_{\lambda(a)} \rightarrow R_{a}$ and a map $h_{a}: R_{a} \rightarrow S_{a}$ such that $g_{a}=h_{a} \circ f_{a}$. Let 
$b \subsetneq a$. Let $p_{b}^{a}: S_{a} \rightarrow R_{b}$ be the projection. We define the map $\phi_{b}^{a}: R_{a} \rightarrow R_{b}$ as $\phi_{b}^{a}=p_{b}^{a} \circ h_{a}$. Then we have

(5) $\phi_{b}^{a} \circ f_{a}=f_{b} \circ \pi_{\lambda(b)}^{\lambda(a)}$.

By (5), (3) is satisfied. Moreover, by (5), we obtain $\phi_{c}^{b} \circ \phi_{b}^{a} \circ f_{a}=\phi_{c}^{a} \circ f_{a}$ for each $c \subset b$. Since $f_{a}$ is "onto", (4) is satisfied. Thus, we have inductively accomplished the desired construction.

By (4), $\left\{R_{a}, \phi_{b}^{a}, A^{*}\right\}$ is an inverse system. For each $a \in A^{*}, R_{a}, \tilde{f}_{a}$ and $\phi_{b}^{a}$ satisfy (a) of Lemma 1. Put $A_{0}=\left\{a \in A^{*}:|a|=1\right\}$. Moreover, let $V_{a}=V_{\alpha}$ for each $a=(\alpha) \in A_{0}$. By (1) and (2), we have

$$
\left\{\tilde{f}_{a}^{-1}\left(V_{a}\right): a \in A_{0}\right\}=\left\{\pi_{\lambda_{\alpha}}^{-1}\left(U_{\alpha}\right): \alpha \in A\right\} .
$$

Hence (b) of Lemma 1 is satisfied. Thus all the conditions of Lemma 1 are satisfied, which concludes $\operatorname{dim} X \leq n$. The proof is complete.

4. Cylindrical limits of inverse systems. Here, we show two propositions which give different kinds of cylindrical limits of inverse systems. Our theorem and these propositions immediately yield two earlier results as corollaries. First, we show

Proposition 1. Let $X=\varliminf_{\lim }\left\{X_{\lambda}, \pi_{\mu}^{\lambda}, \Lambda\right\}$ be the limit of an inverse system of paracompact spaces and perfect maps. Then it is cylindrical.

Proof. We can assume that each $\pi_{\lambda}$ and $\pi_{\mu}^{\lambda}$ is "onto". Since each $\pi_{\mu}^{\lambda}$ is perfect, so is each $\pi_{\lambda}$. Pick a $\lambda_{0} \in \Lambda$ and fix it. Let $\mathcal{U}$ be any open cover of $X$. We can assume $U=\left\{\pi_{\lambda_{\alpha}}^{-1}\left(U_{\alpha}\right): \alpha \in A\right\}$, where $U_{\alpha}$ is a cozero-set in $X_{\lambda_{\alpha}}$ for each $\alpha \in A$. Pick an $x \in X_{\lambda_{0}}$. Since $\pi_{\lambda_{0}}^{-1}(x)$ is compact, we can choose a finite subset $A(x)$ of $A$ such that $\pi_{\lambda_{0}}^{-1}(x) \subset \bigcup_{\alpha \in A(x)} \pi_{\lambda_{\alpha}}^{-1}\left(U_{\alpha}\right)$. Since $\pi_{\lambda_{0}}$ is closed, we can take up an open neighborhood $W_{x}$ of $x$ in $X_{\lambda_{0}}$ such that

$$
\pi_{\lambda_{0}}^{-1}(x) \subset \pi_{\lambda_{0}}^{-1}\left(W_{x}\right) \subset \bigcup_{\alpha \in A(x)} \pi_{\lambda_{\alpha}}^{-1}\left(U_{\alpha}\right) .
$$

Since $X_{\lambda_{0}}$ is paracompact, there exists a locally finite cozero refinement $\mathcal{G}$ of $\left\{W_{x}: x \in X_{\lambda_{0}}\right\}$. Pick a $G \in \mathcal{G}$. Take $p_{G} \in X_{\lambda_{0}}$ with $G \subset W_{p_{G}}$. We choose a $\lambda_{G} \in \Lambda$ with $\lambda_{G} \geq \sup \left\{\lambda_{\alpha}: \alpha \in A\left(p_{G}\right)\right\}$ and $\lambda_{G} \geq \lambda_{0}$. Let

$$
\mathcal{V}(G)=\left\{\left(\pi_{\lambda_{0}}^{\lambda_{G}}\right)^{-1}(G) \cap\left(\pi_{\lambda_{\alpha}}^{\lambda_{G}}\right)^{-1}\left(U_{\alpha}\right): \alpha \in A\left(p_{G}\right)\right\} .
$$

Then we can verify that $\left\{\pi_{\lambda_{G}}^{-1}(V): V \in \mathcal{V}(G)\right.$ and $\left.G \in \mathcal{G}\right\}$ is a locally finite refinement of $U$ by cozero cylinders in $X$. Hence $X$ is cylindrical. The proof is complete.

RemarK 1. Pasynkov $\left[\mathbf{P}_{\mathbf{1}}\right]$ announced a similar result to Proposition 1 without the proof.

Corollary $1\left[\mathbf{K}, \mathbf{P}_{\mathbf{1}}\right]$. Let $X=\varliminf_{\longleftarrow}\left\{X_{\lambda}, \pi_{\mu}^{\lambda}, \Lambda\right\}$ be the limit of an inverse system of paracompact spaces $X_{\lambda}$ such that $\operatorname{dim} X_{\lambda} \leq n$ and perfect maps $\pi_{\mu}^{\lambda}$. Then $\operatorname{dim} X \leq n$.

For the next proposition, we state the following concept which has been introduced earlier in connection with the dimension of the limits of inverse sequences. 
DEFINITION $2\left[\mathbf{N}_{\mathbf{2}}, \mathbf{P}_{\mathbf{2}}\right]$. An inverse sequence $\left\{X_{i}, \pi_{j}^{i}\right\}$ is said to be perforable if for any sequence $\left\{O_{i}\right\}_{i<\omega}$ of open sets $O_{i}$ in $X_{i}$ with $\left(\pi_{j}^{i}\right)^{-1}\left(O_{j}\right) \subset O_{i}, i \geq j$, and $\bigcup_{i<\omega} \pi_{i}^{-1}\left(O_{i}\right)=X$, there exists a sequence $\left\{F_{i}\right\}_{i<\omega}$ of closed sets $F_{i}$ in $X_{i}$ such that $F_{i} \subset O_{i}$ for each $i<\omega$ and $\bigcup_{i<\omega} \pi_{i}^{-1}\left(F_{i}\right)=X$.

The inverse sequences with the perforable property have been enumerated in $\left[\mathbf{N}_{\mathbf{2}}\right.$, Remark 4.2] and [ $\mathbf{P}_{\mathbf{2}}$, Corollary 1].

Proposition 2. If an inverse sequence $\left\{X_{i}, \pi_{j}^{i}\right\}$ of normal spaces $X_{i}$ is perforable, then the limit $X$ is cylindrical.

PROOF. Let $\left\{G_{k}\right\}_{k \leq m}$ be any finite cozero cover of $X$. For each $i<\omega$ and $k \leq m$, let $U_{i}^{k}$ be the maximal open set in $X_{i}$ with $\pi_{i}^{-1}\left(U_{i}^{k}\right) \subset G_{k}$. Put $O_{i}=$ $\bigcup_{k \leq m} U_{i}^{k}$. Then the sequence $\left\{O_{i}\right\}_{i<\omega}$ satisfies the conditions of Definition 2. By the assumption, there exists a sequence $\left\{F_{i}\right\}_{i<\omega}$ of closed sets $F_{i}$ in $X_{i}$, described in Definition 2. Since each $X_{i}$ is normal, there exists a sequence $\left\{C_{i}^{k}: i<\omega\right.$ and $k \leq m\}$ of cozero-sets $C_{i}^{k}$ in $X_{i}$ such that $C_{i}^{k} \subset U_{i}^{k}$ and $F_{i} \subset \bigcup_{k \leq m} C_{i}^{k}$ for each $i<\omega$ and $k \leq m$. Then $\left\{\pi_{i}^{-1}\left(C_{i}^{k}\right): i<\omega\right.$ and $\left.k \leq m\right\}$ is a countable refinement of $\left\{G_{k}\right\}_{k \leq m}$ by cozero cylinders in $X$. Hence $X$ is cylindrical. The proof is complete.

Corollary $2\left[\mathbf{N}_{\mathbf{2}}, \mathbf{P}_{\mathbf{2}}\right]$. Let $X=\lim _{\longleftarrow}\left\{X_{i}, \pi_{j}^{i}\right\}$ be the limit of an inverse sequence of normal spaces $X_{i}$ with $\operatorname{dim} X_{i} \leq n$. If the sequence is perforable, then $\operatorname{dim} X \leq n$.

5. Cylindrical Cartesian products. Here, each Cartesian product is an infinite one unless otherwise stated. Note that a Cartesian product $X$ is represented as the limit of the inverse system of all finite subproducts of $X$ and their projections. So we adopt the following definition for convenience.

Definition 3. A Cartesian product $X=\prod_{\lambda \in \Lambda} X_{\lambda}$ is said to be cylindrical if each finite cozero cover of $X$ has a $\sigma$-locally finite refinement by sets of the form $\pi_{\xi}^{-1}(U)$, where $\xi \subset \Lambda$ with $|\xi|<\aleph_{0}$ and $U$ is a cozero-set in $X_{\xi}$. Moreover, such a set $\pi_{\xi}^{-1}(U)$ is said to be a cozero cylinder in $X$.

By the Theorem, we immediately obtain

COROLlaRY 3. If a Cartesian product $X=\prod_{\lambda \in \Lambda} X_{\lambda}$ is cylindrical, then

$$
\operatorname{dim} X=\sup \left\{\operatorname{dim} X_{\xi}: \xi \subset \Lambda \text { with }|\xi|<\aleph_{0}\right\} .
$$

Now, we consider when a Cartesian product is cylindrical. We prepare two lemmas concerning Cartesian products of paracompact $\Sigma$-spaces.

LEMMA 2. Let $X_{i}$ be a paracompact $\Sigma$-space for each $i<\omega$. Then each open cover of $\prod_{i<\omega} X_{i}$ has a $\sigma$-locally finite refinement by cozero cylinders.

Lemma 2 can be obtained by a modification of the proofs from $\left[\mathbf{N}_{\mathbf{1}}\right.$, Theorems 3.6 and 3.13]. Moreover, Lemma 2 coupled with the proof of $[\mathbf{Y}$, Theorem 1] essentially yields the following result.

LeMma 3. Let $X=\prod_{\lambda \in \Lambda} X_{\lambda}$ be a Cartesian product of paracompact $\Sigma$-spaces, each finite subproduct of which has countable tightness. Let $\Sigma$ be a $\Sigma$-product of $\left\{X_{\lambda}\right\}_{\lambda \in \Lambda}$. Then, for all disjoint closed sets $A$ and $B$ in $\Sigma$, there exists a $\sigma$-locally 
finite cover $\mathcal{U}$ of $X$ by cozero cylinders in $X$ such that each member of $\mathcal{U}$ is disjoint from $A$ or $B$.

PROPOSITION 3. A Cartesian product of paracompact $\Sigma$-spaces, each finite subproduct of which has countable tightness, is cylindrical.

ProOF. Let $X=\prod_{\lambda \in \Lambda} X_{\lambda}$ be a Cartesian product as the above and $\Sigma$ a $\Sigma$ product of $\left\{X_{\lambda}\right\}_{\lambda \in \Lambda}$. Let $G$ be any cozero-set in $X$. We take a sequence $\left\{Z_{i}\right\}_{i<\omega}$ of zero-sets in $X$ such that $G=\bigcup_{i<\omega} Z_{i}$. Fix an $i<\omega$. By Lemma 3, there exists a $\sigma$-locally finite cover $U_{i}$ of $X$ by cozero cylinders in $X$ such that each $U \in U_{i}$ is disjoint from $\Sigma \backslash G$ or $Z_{i} \cap \Sigma$. Since $X \backslash G$ and $Z_{i}$ are closed $G_{\delta}$-sets in $X$, by [PP, Lemma 2], we have

$$
X \backslash G=\mathrm{Cl}(\Sigma \backslash G) \text { and } Z_{i}=\mathrm{Cl}\left(Z_{i} \cap \Sigma\right),
$$

where $\mathrm{Cl}$ denotes the closure in $X$. We set

$$
U_{i}^{*}=\left\{U \in U_{i}: U \cap(\Sigma \backslash G)=\emptyset\right\} .
$$

Then each $U \in \mathcal{U}_{i}^{*}$ and $U \in \mathcal{U}_{i} \backslash \mathcal{U}_{i}^{*}$ are disjoint from $X \backslash G$ and $Z_{i}$, respectively. Since $U_{i}$ is a cover of $X$, we have $Z_{i} \subset \bigcup\left\{U: U \in \mathcal{U}_{i}^{*}\right\} \subset G$. So we obtain $G=\bigcup\left\{U: U \in \mathcal{U}_{i}^{*}\right.$ and $\left.i<\omega\right\}$. Thus $G$ is a $\sigma$-locally finite union of cozero cylinders in $X$. Hence $X$ is cylindrical. The proof is complete.

REMARK 2. For a Cartesian product $X$ as in Proposition 3, it follows from [PP, Proposition 2], [Tk, Theorem 1] and [Y, Theorem 1] that each open $F_{\sigma}$-set in $X$ is a cozero-set. So, Proposition 3 is a generalization of $[\mathbf{K 1}$, Theorem 1].

Proposition 4. Let $X=\prod_{\lambda \in \Lambda} X_{\lambda}$ be a Cartesian product of regular spaces. Then $X$ is cylindrical if one of the following properties is true:

(a) Each countable subproduct of $X$ is Lindelöf.

(b) Each finite subproduct of $X$ is hereditarily Lindelöf.

Proof. First, assume that each finite subproduct of $X$ is Lindelöf. Let $G$ be any cozero-set in $X$. We take a map $g: X \rightarrow[0,1]$ such that $g^{-1}((0,1])=G$. By $\left[\mathbf{E}\right.$, Theorem 1], there exist a $\Gamma \subset \Lambda$ with $|\Gamma| \leq \aleph_{0}$ and a map $f: X_{\Gamma} \rightarrow[0,1]$ such that $g=f \circ \pi_{\Gamma}$. Let $U=f^{-1}((0,1])$.

Case of (a). Since $U$ is an $F_{\sigma}$-set of a Lindelöf space $X_{\Gamma}$, so is $U$. Hence we can choose a sequence $\left\{V_{i}\right\}_{i<\omega}$ of cozero cylinders in $X_{\Gamma}$ such that $U=\bigcup_{i<\omega} V_{i}$. Then $H_{i}=\pi_{\Gamma}^{-1}\left(V_{i}\right)$ is a cozero cylinder in $X$ for each $i<\omega$. One can easily verify $G=\bigcup_{i<\omega} H_{i}$.

Case of (b). Let $\Gamma=\left\{\lambda_{1}, \lambda_{2}, \ldots\right\}$ and $\xi_{i}=\left\{\lambda_{1}, \ldots, \lambda_{i}\right\}$ for each $i<\omega$. Fix an $i<\omega$. Let $W_{i}$ be the maximal open set $X_{\xi_{i}}$ such that $W_{i} \times \prod_{k>i} X_{\lambda_{k}} \subset U$. Since $W_{i}$ is Lindelöf, there exists a sequence $\left\{W_{i j}\right\}_{j<\omega}$ of cozero-sets in $X_{\xi_{i}}$ such that $W_{i}=\bigcup_{j<\omega} W_{i j}$. Then $H_{i j}=\pi_{\xi_{i}}^{-1}\left(W_{i j}\right)$ is a cozero cylinder in $X$ for each $j<\omega$. Here, running $i<\omega$, one can verify $G=\bigcup_{i, j<\omega} H_{i j}$.

In both cases, $G$ is a countable union of cozero cylinders in $X$. Hence $X$ is cylindrical. The proof is complete.

By the Theorem and Propositions 3 and 4, we can obtain some earlier results.

COROLlary 4. Let $X=\prod_{\lambda \in \Lambda} X_{\lambda}$ be a Cartesian product of regular spaces. Then we have

$$
\operatorname{dim} X=\sup \left\{\operatorname{dim} X_{\xi}: \xi \subset \Lambda \text { with }|\xi|<\aleph_{0}\right\}
$$


if one of the following conditions (a)-(c) is satisfied:

(a) Each $X_{\lambda}$ is a paracompact $\Sigma$-space and each finite subproduct $X_{\xi}$ has countable tightness $[\mathbf{Y}]$. In particular, each $X_{\lambda}$ is metrizable $[\mathbf{P o}]$.

(b) Each countable subproduct of $X$ is Lindelöf $[\mathbf{C}, \mathbf{M}]$.

(c) Each finite subproduct $X_{\xi}$ is hereditarily Lindelöf. In particular, it is perfectly normal and Lindelöf $[\mathbf{C}]$.

REMARK 3. Let $S$ be the Sorgenfrey line. Terasawa [T] showed that $\operatorname{dim} S^{\alpha}=0$ for each $\alpha \geq \omega$. Moreover, one can see from his proof that $S^{\alpha}$ is cylindrical for each $\alpha \geq \omega$.

ADDED IN PROOF. Pasynkov [Soviet Math. Dokl. 26 (1982), 654-685] has introduced the concept of piecewise rectangularity for Cartesian products, which is weaker than that of the cylindrical property. Moreover, he has announced that the equality in our Corollary 3 is true for a Cartesian product which is piecewise rectangular.

\section{REFERENCES}

[C] M. G. Charalambous, The dimension of inverse limits, Proc. Amer. Math. Soc. 58 (1976), 289-295.

[E] R. Engelking, On functions defined on Cartesian products, Fund. Math. 59 (1966), 221-231.

[K] Y. Katuta, On the covering dimension of inverse limits, Proc. Amer. Math. Soc. 84 (1982), 588-592.

[K1] B. S. Klebanov, On subspaces of products of metric spaces, Soviet Math. Dokl. 21 (1980), 303-306.

[M] K. Morita, On the dimension of the product of Tychonoff spaces, General Topology Appl. 3 (1973), 125-133.

$\left[\mathbf{N}_{1}\right]$ K. Nagami, $\Sigma$-spaces, Fund. Math. 65 (1969), 169-192.

$\left[\mathbf{N}_{\mathbf{2}}\right] \ldots$, Dimension of non-normal spaces, Fund. Math. 109 (1980), 113-121.

$\left[\mathbf{P}_{1}\right]$ B. A. Pasynkov, On the dimension of products of normal spaces, Soviet Math. Dokl. 14 (1973), $530-533$.

$\left[\mathbf{P}_{\mathbf{2}}\right] \ldots$, On the dimension of topological products and limits of inverse sequences, Soviet Math. Dokl. 22 (1980), 596-601.

$\left[\mathbf{P}_{3}\right] \ldots$ Factorization theorems in dimension theory, Russian Math. Surveys 36 (1981), 175-209.

[Po] E. Pol, On the dimension of the product of metrizable spaces, Bull. Acad. Polon. Sci. 26 (1978), 525-534.

[PP] R. Pol and E. Pol, Remarks on Cartesian products, Fund. Math. 93 (1976), 57-69.

[T] J. Teresawa, On the zero-dimensionality of some non-normal product spaces, Sci. Rep. Tokyo Kyoiku Daigaku Sect. A 11 (1972), 95-102.

[Tk] M. G. Tkačenko, The notion of o-tightness and C-embedded subspaces of products, Topology Appl. 15 (1983), 93-98.

[Y] Y. Yajima, On $\Sigma$-products of $\Sigma$-spaces, Fund. Math. 123 (1984), 49-57.

Department of Mathematics, Kanagawa UniVersity, Yokohama 221, Japan 